Man and Nature

L'homme et la nature

\title{
La valeur sémantique du Nord, du Midi, de l'Orient, et de l'Occident chez Voltaire
}

\section{Krystyna Piechura}

Volume 6, 1987

URI : https://id.erudit.org/iderudit/1011869ar

DOI : https://doi.org/10.7202/1011869ar

Aller au sommaire du numéro

Éditeur(s)

Canadian Society for Eighteenth-Century Studies / Société canadienne d'étude du dix-huitième siècle

ISSN

0824-3298 (imprimé)

1927-8810 (numérique)

Découvrir la revue

Citer cet article

Piechura, K. (1987). La valeur sémantique du Nord, du Midi, de l'Orient, et de l'Occident chez Voltaire. Man and Nature / L'homme et la nature, 6, 45-53.

https://doi.org/10.7202/1011869ar

Copyright (c) Canadian Society for Eighteenth-Century Studies / Sociéte canadienne d'étude du dix-huitième siècle, 1987
Ce document est protégé par la loi sur le droit d'auteur. L'utilisation des services d'Érudit (y compris la reproduction) est assujettie à sa politique d'utilisation que vous pouvez consulter en ligne.

https://apropos.erudit.org/fr/usagers/politique-dutilisation/ 


\section{LA VALEUR SÉMANTIQUE DU NORD, DU MIDI, DE L'ORIENT, ET DE L'OCCIDENT CHEZ VOLTAIRE}

Dans ses discours littéraire, historique et politique, Voltaire a souvent fait référence à des divisions nord-sud et est-ouest de l'Eurasie, distinguant tantôt le Nord du Midi, tantôt l'Orient de l'Occident. Mais l'examen de son oeuvre révèle que ces divisions ne recouvrent pas toujours la même réalité et qu'elles fluctuent en fonction de critères historicogéographiques que nous allons analyser ici. Il existe trois divisions nordsud et est-ouest de l'Eurasie dans les écrits de Voltaire. Elles correspondent à trois types de concepts que nous appellerons "historiques", "contemporains" et "géographiques". Voltaire fait référence à ses concepts "historiques" en parlant d'époques passées, particulièrement de l'antiquité et du Moyen Âge. Quand il écrit sur les événements politiques, sociaux et culturels survenus de son vivant, il mentionne ses concepts "contemporains" de Nord, de Midi, d'Orient et d'Occident. On trouve les concepts "géographiques" voltairiens dans les textes du philosophe qui divisent l'Eurasie suivant des critères purement géographiques.

\section{LE NORD ET LE MIDI}

Les concepts "historiques" de Nord et de Midi, bien expliqués dans l'Essai sur les moeurs, sont les plus larges. Ils découlent de la théorie vol- 
tairienne des migrations d'après laquelle le Nord belliqueux, impitoyable et primitif, a autrefois conquis le Midi plus civilisé mais moins puissant. Selon Voltaire, convaincu que toutes les conquêtes ont été entreprises par le Nord contre le Midi, cette théorie explique les migrations survenues dans le monde entier. La supériorité du Nord se justifie ainsi:

le Nord est plus fécond en hommes vigoureux, capables des longues fatigues, et patients dans les travaux, que les peuples du Midi, occupés de processions, énervés par le luxe, et affaiblis.

Des peuples chasseurs, toujours armés, doivent nécessairement subjuguer des laboureurs et des pasteurs, occupés toute l'année de leurs travaux continuels et pénibles dans leurs foyers. ${ }^{1}$

L'auteur a appliqué ce large concept "historique" de Nord à plusieurs régions du globe et il a utilisé les signifiants Nord et Midi pour nommer de nombreux territoires européens. ${ }^{2}$

Voltaire a souvent fait référence à ses concepts "contemporains" de Nord et de Midi. Cet emploi se retrouve déjà dans l'Histoire de Charles XII, où tous les pays directement ou indirectement engagés dans la guerre du Nord (1700-1721) sont nommés Nord, tandis que les états engagés dans les guerres de Louis XIV sont appelés Midi. Dans les écrits voltairiens, Nord embrasse en général les Royaumes de Suède, du Danemark et de Prusse, le Royaume-Uni de Grande-Bretagne, les Républiques des Provinces-Unies et de Pologne-Lituanie, les états protestants du Saint Empire romain ainsi que l'Empire de Russie. ${ }^{3}$

Voltaire avance l'idée qu'au dix-huitième siècle le Nord, jadis arriéré et barbare, a surpassé le Midi dans tous les domaines de la vie sociale, politique et culturelle:

C'était ... une opinion établie chez les Grecs, que la sagesse viendrait de l'Orient, tandis que, sur les bords de l'Euphrate et de l'Indus, on disait qu'elle viendrait d'Occident. On l'a toujours attendue. Enfin, elle arrive du Nord; elle vient nous éclairer ... elle s'appuie sur la tolérance ... suivie de la paix. ${ }^{4}$

Soulignant que le Nord "contemporain" a non seulement adopté mais aussi enrichi l'héritage romain et grec transmis par le Midi, Voltaire conclut que "l'Éclaircissement vient du Nord". Il est donc évident que de nombreux énoncés voltairiens sur le Nord ont été écrits pour diffuser les idées propagandistes du philosophe, qui a souvent appliqué à tout 
le Nord ses observations basées sur l'exemple d'un seul pays, ou vice versa. Voltaire disait que les changements progressistes avaient été encouragés par "les philosophes sur les trônes". ${ }^{5}$ Selon lui, le Midi (c'està-dire les monarchies du Portugal, d'Espagne, de France, les états italiens et les états catholiques du Saint Empire romain) ne pouvait plus aspirer à jouer un rôle de leader intellectuel de l'Europe - et par conséquent de l'humanité - principalement à cause de son église catholique réactionnaire et intolérante. ${ }^{6}$

Si Voltaire classifie un pays comme faisant partie du Nord "contemporain", cela signifie qu'il le considère comme relativement arriéré du Moyen Âge aux dix-septième ou dix-huitième siècles et qu'il veut souligner, non sans exagération, l'importance des réformes introduites de son vivant. En associant un pays à son concept "contemporain" du Midi, Voltaire indique qu'il le place parmi les états européens qui se sont le mieux développés avant le début du dix-huitième siècle. Puisque certains succès attribués au Nord par Voltaire n'étaient que le produit de son imagination, une analyse de son interprétation du développement de ce territoire nous permettra d'illustrer la façon dont l'un des philosophes du siècle des Lumières se représentait l'Europe de son temps et, ce qui est encore plus important, comment il voulait la réformer.

Dans les contextes géographiques, Voltaire emploie le signifiant Nord pour désigner des zones climatiques arctique et subarctique et le signifiant Midi pour désigner les territoires subtropicaux. ${ }^{7}$

\section{L'OCCIDENT ET L'ORIENT}

Voltaire associe ses concepts "historiques" d'Occident et d'Orient aux territoires asiatiques, africains et européens influencés par les civilisations antiques. La frontière entre l'Orient et l'Occident coïncide avec la frontière entre l'Empire romain de l'est et celui de l'ouest, à cette restriction près que l'Afrique du nord-ouest est située en Orient. L'auteur de l'Essai sur les moeurs croyait que l'Orient était plus vieux que l'Occident et méritait plus d'attention qu'il n'en avait reçu auparavant:

En vous instruisant en philosophe de ce qui concerne ce globe, vous portez d'abord votre vue sur l'Orient, berceau de tous les arts, et qui a tout donné à l'Occident. 
Les climats orientaux, voisins du Midi, tiennent tout de la nature; et nous, dans notre Occident septentrional, nous devons tout au temps, au commerce, à l'industrie tardive. Des forêts, des pierres, des fruits sauvages, voilà tout ce qu'a produit naturellement l'ancien pays des Celtes, des Allobroges, des Pictes, des Germains, des Sarmates, des Scythes ... Les pays fertiles furent les premiers peuplés, les premiers policés. Tout le Levant, depuis la Grèce jusqu'aux extrémités de notre hémisphère, fut longtemps célèbre avant que nous en sussions assez pour connaître que nous étions barbares. ${ }^{8}$

Par conséquent, le discours voltairien suit de près l'expansion des civilisations antiques en Afrique du Nord, en Asie et en Europe. Dans le premier cas, l'Orient inclut toute l'Afrique du Nord décrite comme plus "civilisée" que "l'intérieur de l'Afrique". Quand Voltaire fait référence à l'Asie, l'Orient s'étend jusqu'à la "Tartarie", non délimitée et classifiée comme "septentrionale" et "orientale". Selon l'auteur, l'expansion des cultures "orientales" et "occidentales" dans le Nord n'est pas en contradiction avec sa théorie des conquêtes nord-sud, puisque les conquérants "septentrionaux" barbares assimilaient souvent la culture plus raffinée des peuples qu'ils avaient subjugués - "les Tartares-Mogols", habitants de "la Tartarie", en étant le meilleur exemple. En mentionnant l'Europe, Voltaire insiste sur le fait que la division du pouvoir entre "l'empereur d'Orient" et "l'empereur d'Occident" a engendré en Europe la création de "l'empire d'Orient" et de "l'empire d'Occident" suivie par l'élaboration de deux traditions culturelles respectives. ${ }^{9}$

L'Orient "contemporain" de Voltaire comprend l'Europe du Sud-Est, l'Afrique du Nord et l'Asie entière à l'exception de sa partie nord, et il s'étend à l'ouest jusqu'à l'Empire ottoman. L'Occident inclut l'Italie, la France, l'Angleterre et la Russie, où il atteint sa limite orientale. ${ }^{10}$

Chaque fois que Voltaire mentionne le concept "historique" de l'Occident, le Midi est supérieur au Nord; quand il fait référence au concept "contemporain" de l'Occident, c'est le Nord qui domine le Midi. Méprisant le Midi "contemporain" arriéré, Voltaire s'associe avec le Nord "contemporain". Tout comme sa division nord-midi, la division estouest lui permet de faire ses synthèses historiques et d'utiliser ses observations concrètes concernant un pays pour soutenir ses constatations sur "tout l'Orient" ou "tout l'Occident". ${ }^{11}$

Les concepts "géographiques" voltairiens de l'Orient et de l'Occident sont les plus larges et les plus simples, puisque l'auteur divise l'Eurasie de telle manière que l'Europe se trouve en Occident et l'Asie en Orient. ${ }^{12}$

Ayant élaboré des concepts flexibles sur le Nord, le Midi, l'Orient et l'Occident, Voltaire divise rarement l'Eurasie selon ses parties géographiques (septentrionale, méridionale, orientale et occidentale). Quand 
il le fait, son utilisation de ces divisions géographiques traditionnelles n'est pas toujours précise. ${ }^{13}$

Les divisions voltairiennes de l'Eurasie en Nord, Midi, Orient et Occident ne sont basées que partiellement sur ses observations géographiques; elles émanent bien plutôt de ses constatations philosophiques sur le développement des civilisations humaines. Considéré spécialiste dans le domaine des affaires de l'Eurasie, Voltaire a utilisé ses connaissances de ce continent pour soutenir ses généralisations.

Les divisions ci-dessus ont été profondément influencées par la modification voltairienne de la théorie des climats. Voltaire souligne à plusieurs reprises que son attitude envers cette théorie provient de deux différents modes de pensée: il se considérait à la fois comme élève des écrivains qui s'intéressaient à l'influence du climat sur le développement des gouvernements et des religions (tels Diodore de Sicile, J. Bodin et Montesquieu) et comme disciple des écrivains qui soulignaient l'influence du climat sur le développement de la littérature et des sciences (tels J. Chardin, Y.B. Dubos et Fontenelle). Voltaire indique néanmoins qu'il était loin du prétendu déterminisme géographique de Montesquieu, aussi bien que de la conviction de Fontenelle que les sciences et la littérature ne peuvent fleurir ni dans "la zone torride", ni dans "les deux glaciales", puisque les montagnes Atlas et la mer Baltique constituent les frontières naturelles des territoires "civilisés":

Tout change dans les corps et dans les esprits. Peut-être un jour les Américains viendront enseigner les arts aux peuples de l'Europe.

Le climat a quelque puissance, le gouvernement cent fois plus; la religion jointe au gouvernement encore davantage. ${ }^{14}$

Cette citation aborde le principal problème de la théorie des climats: quels facteurs influencent le développement des cultures humaines? Au dix-huitième siècle, les écrivains divisaient ces facteurs entre causes physiques et morales, concernant respectivement le corps et l'esprit. Le débat centré sur le rôle de ces causes a mené à l'élaboration de nouvelles théories dans le domaine de l'esthétique, des sciences de l'homme, de la philosophie et des sciences naturelles. 
Les doctrines esthétiques sont liées à la querelle des Anciens et des Modernes, puisque certains partisans des Anciens niaient l'universalité de la nature humaine et soulignaient que pour juger les écrivains anciens il fallait les placer dans leur contexte géographico-historique. Lorsque le débat s'est étendu aux cultures contemporaines, Dubos a expliqué sa célèbre théorie esthétique dans ses Réflexions critiques sur la poésie et la peinture (1719). Scientifique et impressionniste, cette théorie suggère qu'en dépit du fait que le sentiment est supérieur à la raison dans les questions de beauté, les causes physiques ont, elles aussi, un rôle à jouer. Suivant des modèles classiques, il nie aussi l'existence du progrès dans les arts. Les adhérents de ce relativisme ont essayé de l'employer dans leurs différentes théories. Voltaire était non seulement au courant des principaux écrits abordant ces problèmes esthétiques, mais il s'était familiarisé aussi avec de nombreuses publications concernant l'application de la théorie des climats aux sciences humaines. Ce sujet avait été étudié par $\mathrm{A}$. de La Mottraye, $\mathrm{C}$. de La Condamine, P. de Maupertuis, et $\mathrm{H}$. de Boulainvillier dans leurs descriptions de nations ou tribus particulières; ainsi que par l'abbé de Saint-Pierre, l'abbé $d$ 'Espiard et $P$. Huet dans leurs considérations théoriques ou philosophiques. Voltaire a même consulté des publications spécialisées sur la nature de l'air, écrites par S. Hales, J. Privat de Molières et N. Regnault. ${ }^{15}$

Dans ses polémiques concernant l'influence de l'environnement sur la civilisation, Voltaire désirait modifier les opinions de Montesquieu, auteur de la meilleure version de la théorie des climats publiée durant la première moitié du dix-huitième siècle. Montesquieu liait l'ordre politique d'un état à ses conditions géographiques. Selon lui, les grands empires ont généralement un gouvernement despotique, les états de grandeur moyenne sont des monarchies et les petits états des républiques. L'Esprit des lois, basé sur des renseignements soigneusement compilés et appuyé sur des méthodes d'analyse spécifiques à l'histoire, à la jurisprudence, à la philosophie et aux sciences de l'homme, illustre la coexistence des causes physiques et morales. L'oeuvre de Montesquieu est devenue une source importante pour la géographie, la politique, la philosophie et les coutumes de l'Eurasie. Notons que Voltaire avait raison quand il accusait l'auteur de l'Esprit des lois d'apriorisme, puisque ses méthodes et ses informations avaient été souvent empruntées aux écrivains contemporains et antiques, et avaient été exprimées dans les oeuvres précédentes de Montesquieu, comme les Lettres persanes, l'Essai sur les causes qui peuvent affecter les esprits, les Mémoires sur les mines, les Réflexions sur les habitants de Rome, et les Considérations sur les causes de la grandeur des Romains. La valeur de l'Esprit 
des lois réside donc dans la cohérence de sa théorie qui se veut appuyée sur le raisonnement scientifique et l'expérience pratique. ${ }^{16}$

Bien que la théorie des climats ait ainsi reçu une confirmation scientifique de la part d'un grand nombre de critiques, Voltaire, très pragmatique, a questionné la véracité des observations de Montesquieu. À plusieurs reprises, il a utilisé son savoir dans les domaines des sciences humaines, sociales et naturelles pour corriger les erreurs prétendues ou réelles de son illustre prédécesseur. Dans ses critiques acharnées et souvent exagérées, Voltaire accuse Montesquieu, à tort, de déterminisme géographique et se rapproche du relativisme - il souligne que seuls les peuples primitifs sont totalement dépendants de l'environnement, puisque plus le niveau de développement d'une nation est élevé, moins celle-ci dépend de la nature. ${ }^{17}$

L'environnement joue cependant un rôle dans la théorie de la causalité de Voltaire, un des points les plus faibles de sa méthodologie de l'histoire. L'auteur croyait qu'une fois que Dieu avait fini son oeuvre de créateur, l'univers s'était mis à fonctionner de lui-même. Ignorant le rôle de la Providence, Voltaire se trouvait en face d'un problème important: déterminer le fonctionnement des facteurs responsables du développement de l'humanité. Pour lui, la société humaine est la conséquence d'une série de facteurs internes et externes. Il place au premier plan l'ordre socio-politique, la culture, la religion, le rôle des grands hommes et l'amour naturel pour la justice et l'ordre. Au second plan se trouve l'environnement, c'est-à-dire le climat, la fertilité de la terre, le réseau fluvial, la proximité de bords de mers et la configuration du terrain. Il insiste sur le fait que ces facteurs ont été formés par les forces mécaniques de l'univers, mais qu'ils pouvaient être transformés grâce aux efforts humains. En plus de ces deux groupes de facteurs, il cite "le hasard", auquel il se réfère quand il ne peut pas trouver de meilleure explication pour l'origine d'un événement. ${ }^{18}$

Voltaire croyait que le développement culturel, politique, social et économique d'un pays était influencé mais pas déterminé par l'environnement géographique. Désireux de systématiser, de répandre, et de développer la civilisation en Eurasie, il avait besoin d'une terminologie nouvelle qui lui aurait permis d'expliquer ses conceptions et de présenter ses conclusions d'une manière facile et claire. Ayant écrit sur le sujet pendant presque toute sa longue vie, il a souvent changé d'idées. Néanmoins, comme nous l'avons montré, il se perdait parfois dans ses propres conclusions et était imprécis et négligent. Les quatre signifiants - Nord, Midi, Orient et Occident - correspondent à plusieurs signifiés. Dans les pages de son Histoire de l'empire de Russie, le lecteur peut trouver deux concepts différents du Nord, et le plus 
patient des étudiants de son Essai sur les moeurs ne pourrait ni situer l'Orient ni en identifier les habitants.

Les divisions voltairiennes de l'Eurasie doivent être placées dans les courants des deux écoles qui dominaient les sciences humaines pendant la première moitié du dix-huitième siècle: les écoles naturaliste et historico-philologique. La première s'intéressait à l'analyse des lois de la nature et de la religion naturelle, et postulait que la nature humaine avait des traits universels et permanents. La seconde école, moins dogmatique, cherchait des arguments historiques qui auraient conduit à des généralisations concernant certains développements culturels sur des territoires spécifiques à des époques données. Comme les arguments, les méthodes et les idéologies de ces écoles étaient parfois très semblables, les écrivains ont souvent fait référence aux deux modes de pensée.

Puisque nous ne connaissons l'opinion de Voltaire que sur quelques états de l'Eurasie, une analyse de sa façon de diviser ce continent peut nous aider à reconstruire sa vision du progrès, à déterminer les critères d'après lesquels il classifiait certains territoires européens comme "barbares" et d'autres comme "civilisés", et à établir comment, en combattant certains stéréotypes médiévaux ("mensonges" et "préjugés"), il élaborait des stéréotypes modernes qui ont souvent été repris par la suite.

KRYSTYNA PIECHURA

Université de Toronto

\section{NOTES}

1. Fragment des instructions pour le prince royal de ${ }^{* *}$ (1752), M.XXVI.442; Essai sur les moeurs (1754-1758, 1769), M.XI.271, M.XII.446; l'abréviation M. suivie par les numéros de volume et de(s) page(s) indique qu'on cite Voltaire, Oeuvres complètes, éd. L. Moland, 51 vols. (Paris, 1877-1885). Cf. aussi Commentaire sur "l'Esprit des Lois" (1777), M.XXX.444; Best.D (17307), Voltaire à A.P. Shouvalov, 19 juil. 1771, dans: Voltaire, Correspondence and Related Documents. The Complete Works of Voltaire, éd. T. Besterman, vols. 85-135 (Banbury, Oxfordshire, 1968-1976). Cette édition sera dorénavant identifiée comme Best.D.

2. Cf. p.e. Défense du 'Mondain' (1737), M.X.93; Essai sur les moeurs, M.XI.271.

3. Histoire de Charles XII (1731), M.XVI.149, 163, 198, 211, 264, 266, 322-323, 347. 
4. Sermon prêché à Bâle (1768), M.XXVI.582-583; cf. Histoire de Charles XII (1731), M.XVI.149, 163, 198, 211, 264, 266, 322-323, 347; Le Russe à Paris (1760), M.X.127; Histoire de l'empire de Russie (1759-1762), M.XVI.426, 478, 516, 546, 550, 556-558, 561.

5. Dictionnaire philosophique (1771), M.XX.201. Concernant les exemples de ses généralisations cf. Essai sur les moeurs M.XI.275, 287-288, M.XIII.123-125; Histoire de l'empire de Russie, M.XVI.404; Epitre au roi de Suède, Gustave II (1771), M.X.438.

6. Cf. Connaissance des beautés et des défauts de la poésie (1749), M.XXIII.417; Annales de l'Empire (1753-1754), M.XIII.322, 340, 485-486; Essai sur les moeurs, M.XI.275, 287-288, M.XIII.135.

7. Histoire de l'empire de Russie, M.XVI.410, 414.

8. Essai sur les moeurs, M.XI.158-159, cf. aussi M.XI.146, M.XI.478-488.

9. Essai sur les moeurs, M.XI.43, 240-256, 262-265, 327-337, 430-479; XII.79-78, 149-151; Annales de l'Empire, M.XIII.165-167, 237, 253, 344, 446; Des droits des hommes et des usurpations des papes (1768), M.XXVII.194-195.

10. Connaissance des beautés et des défauts de la poésie, M.XXIII.407; Essai sur les moeurs, M.XI.157-164, 478-488; Epitre à Kienlong, roi de la Chine (1770), M.X.412-413; Epitre à l'impératrice de Russie, Catherine II (1771), M.X.435; Best.D 13996, Voltaire à Catherine II, 27 fèv. 1767.

11. Essai sur les moeurs, M.XI.155, 159, M.XII.448; Histoire de l'empire de Russie, M.XVI.526.

12. Essai sur les moeurs, M.XI.478-488, M.XIII.120; Histoire de l'empire de Russie M.XVI.596.

13. Histoire de Charles XII, M.XVI.156; Essai sur les moeurs, M.XIII.127; Histoire de l'empire de Russie, M.XVI.403, 541; Le pyrronisme de l'histoire [1768], M.XXVII.296.

14. Dictionnaire philosophique, M.XVIII.199-200.

15. R. Mercier, “La théorie des climats des 'Réflexions critiques' à 'L'Esprit des lois'," Revue d'histoire littéraire de la France LIII (1953), pp. 17-37, 161-174.

16. R. Mercier, "La théorie des climats des 'Réflexions critiques' à ' $L$ 'Esprit des lois'," p. 31-37, 170-174; F.V. Tarnawsky, "Monteske o Rossii (K istorii 'Nakaza' imperatricy Ekateriny II)," dans: Troudy rousskikh' utchenykh' za-granitsei, éd. A. I. Kaminka, vol. 1 (Berlin, 1922), pp. 209-211, 223.

17. Cf. Essai sur les moeurs, ch. CXCVII; Dictionnaire philosophique, article "Climat", "Lois"; Commentaire sur "l'Esprit des lois" M.XXX.442-445; P. Martino, L'Orient dans la littérature française aux XVIIème et XVIIIème siècles (Paris, 1906), pp. 290-295.

18. J.B. Black, The Art of History (New York, 1965), pp. 37-47. 